\title{
UV-NIR spectra of two planetary nebulae with X-Shooter
}

\author{
Cezary Szyszka ${ }^{1}$, Jeremy R. Walsh ${ }^{2}$ and Albert A. Zijlstra ${ }^{1}$ \\ ${ }^{1}$ Jodrell Bank Centre for Astrophysics, University of Manchester, United Kingdom; ${ }^{2}$ European \\ Southern Observatory, Garching, Germany
}

\begin{abstract}
The ESO VLT spectrograph X-shooter is currently the only one capable of observing the range from $330 \mathrm{~nm}$ up to $2.5 \mu \mathrm{m}$ in a single shot. X-shooter is very suitable for study of the many diagnostic emission lines in PN providing a uniform flux scale at medium resolution over a large instantaneous wavelength range. Spectroscopy of two contrasting PNe, NGC 6302 and M 2-9, were observed and methods explored for identification of the many fitted emission lines.
\end{abstract}

Keywords. planetary nebulae: individual (M2-9, NGC 6302), techniques: spectroscopic

\section{Motivation and Observations}

Quantitative measurement of emission lines from astrophysical ionized media play a fundamental role in PN research and provides a bridge to laboratory astrophysics where processes in such rarefied media are not amenable to experiment. Identification and interpretation of emission lines are fundamental for determination of physical conditions temperature, density - and abundances. The recent trend to deeper and deeper spectra, approaching the sub $10^{-5} F(\mathrm{H} \beta)$ regime, has revealed a wealth of recombination lines of the lighter elements but also forbidden lines of heavier elements beyond Fe, such as elements from rows 4-6 of the Periodic Table (Baluteau et al. 1995; Sharpee et al. 2007), considered to arise in the s-process.

While the accurate reduction of the spectra and the reliable fitting of emission lines is now considered routine (although manually repetitive), the identification of faint lines with atomic databases is still fraught with hurdles. For the common lines of light elements this is less a problem since many template spectra have been published and the excellent Atomic Line List v2.04 of P. van Hoof is available. However for heavier elements and sorting out multiplet members, more sophistication is required. EMILI (Sharpee et al. 2003) provides an automatic tool for aiding the decision making process by ranking the line identifications based on relative intensity, presence of other lines from the same multiplet and velocity shift tied to ionization level. EMILI uses the Atomic Line List as source for the spectral lines, so the heavy elements are not present. Sharpee et al. (2007) provide very deep line lists for several $\mathrm{PNe}$, including many faint recombination lines of $\mathrm{O}, \mathrm{N}$ and $\mathrm{Ne}$.

With the aim of extending the availability of template spectra over a wider range than just the optical, new deep spectra have been obtained in X-shooter Science Verification time of a low ionization PN (M2-9: PNG $010.8+18.0)$ and a very high ionization PN (NGC 6302: PNG 349.5 +01.0). The X-shooter spectrometer consists of three arms, each with optimized optics, échelle grating and detector: UVB (300-559.5 nm, $R \sim 5000$ for $1^{\prime \prime}$ slit); VIS (559.5-1024 nm, $R \sim 8000$; NIR (1024-2480 nm, $R \sim 5200$. Details of the instrument and its operation can be found in Vernet et al. 2007. 


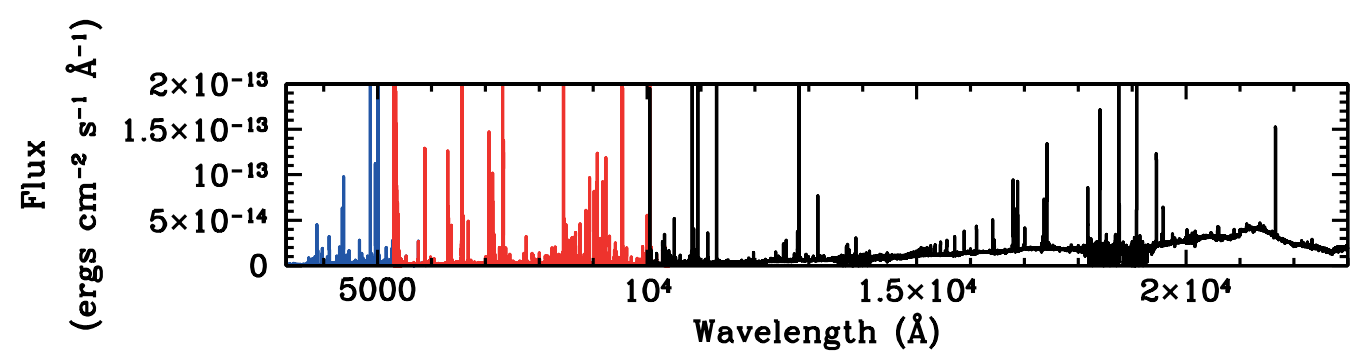

Figure 1. X-shooter spectrum of the core of M2-9 with UVB (blue), VIS (red) and NIR (black) spectra overlaid.

\section{Emission line fitting and identification}

For NGC 6302 the nebular spectrum along the full X-shooter slit length $\left(11 \times 0.5^{\prime \prime}\right)$ was summed and the offset sky subtracted; for M2-9, the bright core only was extracted and the sky spectrum was taken from both ends of the slit. Fig.1 shows the full $(0.33-2.3 \mu \mathrm{m})$ spectrum of M2-9. The signal-to-noise $(\mathrm{S} / \mathrm{N})$ allows detection of lines to $5 \times 10^{-5} \mathrm{~F}(\mathrm{H} \beta)$ in NGC 6302 and $3 \times 10^{-4}$ for M2-9. The broad Raman features at 6830 and $7087 \AA$ (discovered by Groves et al. 2002) were detected in NGC 6302; for M2-9 the strong continuum and a forest of lines, mostly Fe II and Fe III, many listed by Torres-Peimbert et al. (2010) in the optical, are seen. The separate UVB, VIS and NIR spectra were analysed with a code that enables fully automatic, semi-automatic and fully interactive line fitting with multiple Gaussians and various fits to the continuum. For NGC 6302 957 emission lines were fitted and for M2-9 1339 lines for $\mathrm{S} / \mathrm{N}>3$. The typical fit parameters such as wavelength, integrated flux, FWHM, equivalent width and their errors are recorded.

Line identification was performed in two ways - one with the time-consuming match with existing spectra and previous tabulations and another with EMILI. For NGC 6302, the line identifications in Groves et al.(2002), Sharpee et al. (2007) and Baluteau et al. (1995) were used, and identifications manually recorded. For M2-9, the EMILI code was employed for lines up to $11000 \AA$. Sharpee et al. (2003) developed weighting criteria for prioritizing line identifications, but in the existing version of the code do not use the FWHM information and line strengths of lines from different multiplets in arriving at line IDs. Current deficiencies of EMILI as far as this work is concerned are the long wavelength limit of $11000 \AA$ and the lack of an attached database of heavier element lines. The identifications from EMILI (and traditional methods) can be seen as an intermediate step to full spectral synthesis using line lists, available atomic data, density and temperature from diagnostic line ratios and observed velocity to model the lines, as developed by Péquignot and Morrisset (outlined in Péquignot 1996). Only with this comprehensive approach will reliable identification of the weakest and most blended lines be achieved.

\section{References}

Baluteau, J. P., Zavagno, J. P., Morisset, C., et al. 1995, A\&3A, 390, 175

Groves, B., Dopita, M. A., \& Williams, R. E. 2002, PASA, 19, 425

Péquignot, D. 1996, Physica. Scripta T, 65, 137

Sharpee, B., Williams, R. E., Baldwin, J. A., et al. 2003, ApJS, 149, 157

Sharpee, B., Zhang, Y., Williams, R. E., et al. 2007, ApJ, 659, 1265

Torres-Peimbert, S., Arrieta, S., \& Bautista, M. 2010, Rev. Mex. Astr. Astrophys., 46, 221

Vernet, J., D'Odorico, S., Christensen, L., et al. 2007, ESO Messenger, 130, 5 